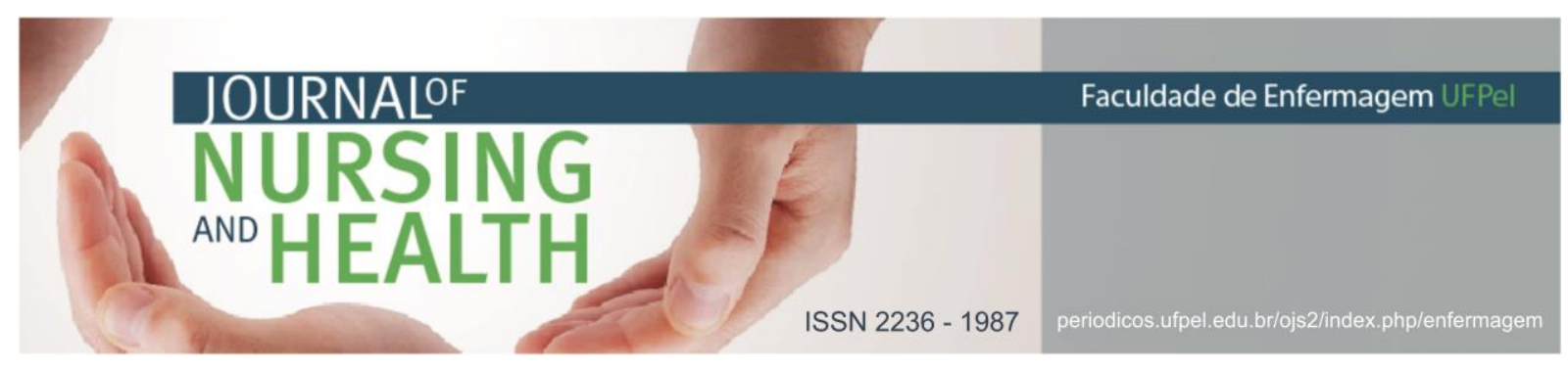

ARTIGO ORIGINAL

\title{
Alimentação e prática de atividades físicas de crianças: hábitos cotidianos e culturais
}

\author{
Food and activities of children's physical practice: daily habits and culture
}

\section{Alimentos y actividades de la práctica física de los niños: hábitos diarios y culturales}

Sebold, Luciara Fabiane ${ }^{1}$; Silva, Paola Pitol da²; Melcher, Tayara Aline ${ }^{3}$; Silveira, Bárbara Mohr da ${ }^{4}$; Justino, Juliana Simas ${ }^{5}$; Girondi, Juliana Balbinot Reis ${ }^{6}$

\section{RESUMO}

Objetivo: conhecer os hábitos cotidianos de alimentação e prática de atividades físicas de crianças entre sete e dez anos de idade, de uma unidade básica de educação de um município do Sul de Santa Catarina. Método: pesquisa qualitativa descritiva e exploratória, desenvolvida com 24 crianças em uma unidade de ensino, vinculada a Estratégia de Saúde da família. Os dados foram coletados no período de outubro de 2012, por meio de entrevista semiestruturada e analisados a luz do referencial teórico de Madeleine Leininger. Resultados: o cotidiano das crianças e de suas famílias nem sempre auxiliam nos hábitos saudáveis, padrões culturais podem influenciar neste processo, consequentemente refletindo no ambiente escolar. Conclusão: parcerias entre a Estratégia de Saúde da Família (ESF), escola e família são importantes para traçar estratégias que fomentam as práticas de educação em saúde desta comunidade.

Descritores: Hábitos alimentares; Atividade Motora; Obesidade; Enfermagem; Criança.

\section{ABSTRACT}

Objective: to learn about the everyday habits of food and physical activity of children from seven to ten years of age, from a basic unit of education at a city in the south of Santa Catarina. Methods: qualitative descriptive and exploratory study, developed with 24 children in a unit of education, linked to the Family Health Strategy. The data was collected in October 2012, through semistructured interviews, and analyzed through the theoretical framework of Madeleine Leininger. Results: the daily life of children and their families do not always assist in healthy habits; cultural patterns may influence this process, consequently reflecting in the school environment. Conclusion: partnerships among the Family Health Strategy (FHS), school, and family are important to devise strategies that foster the practices of health education in this community.

Descriptors: Healthy Diet; Motor Activity; Obesity; Nursing; Child.

\footnotetext{
${ }^{1}$ Enfermeira. Doutora em Enfermagem. Docente do Departamento de Enfermagem Universidade Federal de Santa Catarina, Florianópolis, Santa Catarina, Brasil. E-mail: fabiane.sebold@ufsc.br. http://orcid.org/0000-00025023-9058

2 Enfermeira. Lar de Convivência para Idosos Divino Espírito Santo. Tapejara, Rio Grande do Sul, Brasil. E-mail: pa_olynha@hotmail.com. http://orcid.org/0000-0001-8809-9145

3 Enfermeira. Fundação Hospitalar. Imbuía, Santa Catarina, Brasil. E-mail: tayy-enf@hotmail.com. https://orcid.org/0000-0003-2003-7719

${ }^{4}$ Acadêmica de Enfermagem. Curso de Graduação em Enfermagem da Universidade Federal de Santa Catarina. Florianópolis, Santa Catarina, Brasil. E-mail: barbaramohrs@gmail.com. http://orcid.org/0000-0003-4428-5346

${ }^{5}$ Acadêmica de Enfermagem. Curso de Graduação em Enfermagem da Universidade Federal de Santa Catarina. Florianópolis, Santa Catarina, Brasil. E-mail: jusimasj14@gmail.com. http://orcid.org/0000-0001-7413-8270

${ }^{6}$ Enfermeira. Doutora em Enfermagem. Docente do Departamento de Enfermagem Universidade Federal de Santa Catarina, Florianópolis, Santa Catarina, Brasil. E-mail: juliana.balbinot@ufsc.br. http://orcid.org/0000-0002$3763-4176$
} 


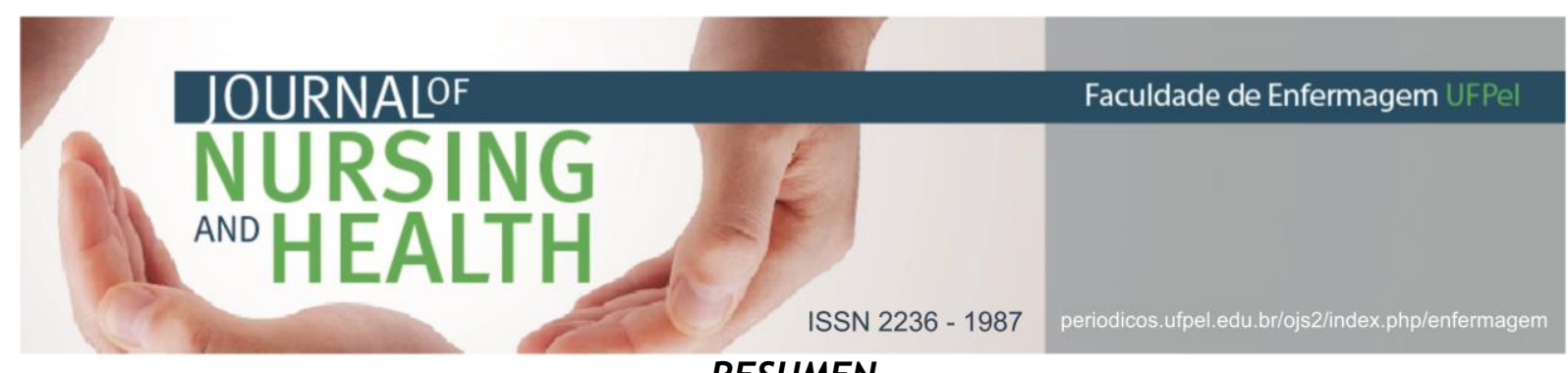

RESUMEN

Objetivo: aprender acerca de los hábitos diarios de alimentación y la actividad física de los niños entre siete y diez años de edad, de una unidad básica de educación de un municipio en el sur de Santa Catarina. Métodos: cualitativo descriptivo y exploratorio, desarrollado con 24 niños en una unidad de educación, vinculados a la Estrategia Salud de la Familia. Los datos fueron recolectados en octubre de 2012, por medio de entrevistas semiestructuradas y analizados baja el marco teórico de Madeleine Leininger. Resultados: la vida cotidiana de los niños y sus familias no siempre ayudan a los hábitos saludables; los estándares culturales pueden influir en este proceso, por consiguiente, que refleja en el entorno escolar. Conclusión: las asociaciones entre la Estrategia de Salud de la Familia (ESF), la escuela y la familia son importantes para diseñar estrategias que fomenten las prácticas de educación para la salud en esta comunidad.

Descriptores: Dieta Saludable; Actividad Motora; Obesidad; Enfermería; Niño.

\section{INTRODUÇÃO}

Os Hábitos saudáveis devem ser cultivados desde a gestação até o envelhecimento. Estes envolvem, dentre outras a práticas de atividade física e alimentação saudável. As estratégias da Política de Promoção da saúde contemplam, dentre outras, ações de incentivo de atividade física e de hábitos saudáveis alimentares. ${ }^{1}$

Uma alimentação saudável deve contemplar as seguintes características ser acessível física e financeiramente, harmônica, ser considerada segura sanitariamente, de aparência colorida, agradável ao paladar, ou seja, saborosa, e principalmente variada quanto os nutrientes. ${ }^{2}$

As ações para a alimentação saudável precisam seguir alguns princípios como: a adequação da quantidade e qualidade; variação de forma a facilitar a oferta de todos os nutrientes necessários ao organismo; segurança, dos pontos de vista sanitário e genético; garantia do acesso físico e financeiro; atrativo do ponto de vista sensorial; respeito à cultura alimentar do indivíduo ou grupo a que se destina. ${ }^{2}$
A não adoção de hábitos como a alimentação saudável e a prática de atividades físicas podem acarretar no em prejuízos a saúde, dentre eles o aumento de peso, ou seja, o sobrepeso e a obesidade. De acordo com a Organização Mundial de Saúde (OMS) entende sobrepeso e obesidade como o acúmulo de gordura anormal ou excessivo que podem prejudicar a saúde. ${ }^{3} \mathrm{Em}$ consequência do excesso de peso podem surgir algumas comorbidades como: doenças cardiovasculares e diabetes Mellitus. Além disso, a obesidade pode causar incapacidade funcional, reduzir a qualidade de vida e aumentar a mortalidade. ${ }^{4}$

Observa-se também, que com as mudanças de hábitos no cotidiano moderno, o aparecimento de um ambiente obesogênico favoreceram poucos hábitos saudáveis, levando as pessoas e, principalmente as crianças ao aumento de peso. Assim, o reconhecimento dos fatores causadores da obesidade favorece a prevenção dos fatores de risco cardiovascular na infância e auxiliam nas intervenções destinadas a melhora 
da saúde e a redução das taxas de morte prematura. ${ }^{5}$

Neste contexto, o enfermeiro deve estabelecer estratégias de saúde, planejando e desenvolvendo de ações que buscam minimizar a incidência de sobrepeso e obesidade na população infantil, visando à promoção da saúde, prevenção de doenças advindas obesidade e, para isso precisa conhecer os hábitos cotidianos das crianças e suas famílias para então, fomentar práticas de educação alimentar e a realização de exercícios físicos.

Diante destes referenciais, o objetivo foi: Conhecer os hábitos cotidianos de alimentação e prática de atividades físicas de crianças entre sete e dez anos de idade, de uma unidade básica de educação de um município do Sul de Santa Catarina.

\section{MÉTODOS}

Estudo qualitativo, descritivo e exploratório. Desenvolvido com 24 crianças, na faixa etária de sete a dez anos de idade em uma escola de ensino básico de um município do sul de Santa Catarina. Os dados foram coletados por meio de entrevista semiestruturada que abordava os hábitos cotidianos relacionados à alimentação e atividades físicas das crianças, as mesmas foram entrevistadas individualmente, em ambiente reservado. A coleta dos dados deu-se em outubro de 2012. As crianças participantes tiveram a permissão dos pais ou responsáveis representados assinaram o Termo de Consentimento Livre e Esclarecido (TCLE).

Foram escolhidos os alunos nessa faixa etária, porque após o levantamento dos dados antropométricos realizados pela Estratégia de Saúde da Família (ESF) do bairro da escola, evidenciaram índice significativo de crianças com sobrepeso/obesidade nesta faixa etária, entretanto vale ressaltar dois aspectos importantes: todas as crianças desta faixa etária participaram do estudo, mesmo não sendo classificadas com sobrepeso e/ou obesidade e, os dados apresentados no quadro 1 foram disponibilizados pela equipe da ESF.

\begin{tabular}{|c|c|c|c|c|c|}
\hline Idade (anos) & $\begin{array}{c}N^{\circ} \text { de } \\
\text { Crianças }\end{array}$ & IMC & Normal & Sobrepeso & Obesidade \\
\hline \multicolumn{7}{|c|}{ Meninos } \\
\hline 7 e 8 & 25 & $<15,4->28,4$ & 18 & 4 & 3 \\
\hline 9 e 10 & 26 & $<16,1->26,7$ & 18 & 7 & 3 \\
\hline \multicolumn{7}{|c|}{ Meninas } \\
\hline 7 e 8 & 18 & $<13,4->26,2$ & 4 & 9 & 5 \\
\hline 9 e 10 & 17 & $<15,5->25,6$ & 8 & 5 & 4 \\
\hline
\end{tabular}

Quadro 1 - Avaliação antropométrica das crianças de 07 a 10 anos de idade em uma escola da região sul de Santa Catarina no ano de 2012

Fonte: ESF/ Estratégia de Saúde da Família Willi Tomio - Bairro Canoas. Rio do Sul/SC/ Brasil, 2012. 


\section{JOURNALOF \\ NURSING \\ ANO HEALTH \\ ISSN 2236 - 1987}

Os critérios de inclusão foram: crianças na faixa etária de sete a dez anos de idade, que estiveram presentes nos dias das atividades educativas sobre hábitos saudáveis desenvolvidas na escola e que tinha o TCLE assinado. Foram excluídos os indivíduos sem TCLE; que não estiveram presentes na instituição no momento da coleta dos dados.

Os dados foram analisados de acordo com a Teoria de Enfermagem Transcultural de Madeleine Leininger ${ }^{6}$ e organizados seguindo a análise de conteúdo de Bardin ${ }^{7}$ que categoriza os mesmos para melhor interpretação dos achados no estudo. As categorias construídas foram: Hábitos alimentares cotidianos e, Hábitos relacionados à prática de atividade física.

0 presente projeto de pesquisa foi aprovado pelo Comitê de Ética de pesquisa com Seres Humanos da UNIDAVI, protocolo $\mathrm{n}^{\circ}$ 981. Foram garantidos a privacidade e o sigilo de suas identidades, sendo utilizados nomes correspondentes a letra " $C$ " de criança seguida pelo algarismo arábico de sua entrevista.

\section{RESULTADOS}

As crianças revelam que os hábitos alimentares em seus cotidianos variam conforme a rotina de suas famílias, ligadas aos hábitos culturais. Em contrapartida, no ambiente escolar, acabam por experimentar um cardápio variado, que nem sempre corresponde às suas preferências alimentares.

\section{Hábitos alimentares cotidianos}

As crianças deste estudo relatam que em suas casas sua alimentação é pouco variada, relatam que a primeira refeição da manhã e, consomem apenas alguns alimentos e que não contemplam frutas e cereais.

O meu café da manhã é com café com pão [...] com mouse com nata (C3).

Como pão com margarina e café [...] de tarde eu tomo água, como pão, chocolate (C8).

De manhã eu como pão $e$ achocolatado [...] pão com açúcar (C9).

Segundo o depoimento de algumas crianças tem o cotidiano familiar atribulado e, por conta disso as acabam não conseguindo realizar a primeira refeição do dia, o café da manhã.

Eu não tenho vontade de comer de manhã (C13).

Eu não tomo café da manhã porque eu acordo atrasado para a escola e sempre tenho que ir rápido (C11).

Hoje eu não tomei café da manhã [...] meu pai está sempre correndo e atrasado (C14).

Eu não tomo café da manhã porque é muito apressado e meus pais tem que trabalhar (C15).

Relacionado à ingestão de frutas e verduras no cotidiano, relatam não 
possuírem o hábito de tê-las em suas refeições diárias.

Não gosto de mamão [...] nem de verdura que tem cebola [...] acho que não gosto de nenhuma verdura (C5).

Não como nada de salada [...] tem gosto ruim e não gosto (C7).

E não gosto de pêssego e uva, porque meu pai não gosta aí eu também não gosto também (C9).

Eu não gosto de frutas (C19).

Não gosto de verdura (C20).

Verdura, não como nenhuma e fruta só às vezes banana (C23).

Durante o período que estão na escola relatam que se alimentam com o lanche oferecido na mesma. Porém, devemos ressaltar que algumas crianças, preferem trazer suas refeições de casa.

Como a comida da escola quando é pão e margarina as outras coisas não gosto (C1).

$\mathrm{Na}$ escola eu como sopa $e$ cachorro quente (C4).

Na escola eu só não como caldo de peixe porque eu não gosto [...] Tem cheiro ruim (C8).

Eu sempre como o lanche da escola, menos segunda feira que tem caldo de peixe (C15).

Segunda-feira eu não como na escola porque tem caldo de peixe (C16).
Apesar de a escola oferecer 0 cardápio, algumas crianças preferem os lanches industrializados.

Trago salgadinho, achocolatado e às vezes eu compro um refrizinho na venda da frente da escola (C11).

Eu trago bolacha para escola porque é só isso que tem em casa (C12).

Eu trago para escola achocolatado, salgadinho um monte de coisa que minha mãe compra no supermercado (C13).

Eu trago para escola bolacha, salgadinho e suco de caixinha (C17).

Eu trago bolacha recheada, salgadinho, refrigerante (C18).

\section{Hábitos relacionados à prática de atividade física}

Em relação ao dia-a-dia das crianças observou-se que a grande maioria prefere atividades que exijam coordenação motora, equilíbrio, força física. Apesar da maioria optar por essas atividades, houve crianças que preferem atividades de pouca movimentação, e as mais citadas são computador e videogame.

Evidencia-se nas falas abaixo, que mesmo com o desenvolvimento das tecnologias, algumas crianças optam por brincadeiras que exijam movimentação corporal.

Eu brinco de pega-pega, futebol no campo próximo da minha casa e na escola (C2). 
Eu gosto de pular corda na hora do recreio (C12).

Eu ando de bicicleta na minha rua com meus irmãos e amigos (C20).

Outros participantes por sua vez citaram a preferência por brincadeiras que não exigem muita movimentação física, e desta forma mantendo-as sedentárias em relação às brincadeiras com movimentação corporal.

Eu fico na frente do computador a tarde porque fico em casa sozinho(C6).

Eu jogo vídeo game a tarde toda (C8).

Em casa eu assisto TV e jogo videogame com meus amigos (C11).

De tarde eu brinco no computador não posso brincar na rua [...] é perigoso (C15).

Assistir um pouco de televisão, mas gosto de jogar vídeo game sempre (C21).

\section{DISCUSSÃO}

Neste estudo as crianças relatam suas opções de alimentares e suas práticas de atividade física e que, por hora, evidenciam práticas não saudáveis. Associações significativas de criança sobrepeso / obesidade com a obesidade dos pais, família comportamentos, hábitos alimentares e atividade física confirmam a complexidade da obesidade infantil, e neste sentido a necessidade de prevenir sobrepeso infantil através de
ISSN 2236 - 1987

medidas que envolvam a família da criança, especialmente relacionados com a adequação do estado nutricional dos pais; a necessidade de mudanças nos hábitos alimentares; e, principalmente, o importância de desencorajar o consumo de produtos ultra-processados. ${ }^{8}$

A praticidade dos alimentos industrializados vem conseguindo maior espaço no cotidiano alimentar das famílias, principalmente aquelas que possuem uma rotina ocupada. Mesmo os pais tendo o conhecimento de que esses alimentos não são saudáveis e não possuírem os nutrientes adequados continuam os oferecendo aos filhos, tendo em vista a comodidade que eles proporcionam. ${ }^{9}$

Os depoimentos coletados neste estudo demonstram que o hábito de realizar a primeira refeição pela manhã pode estar diretamente relacionado tanto ao fator cultural, quanto ao incentivo familiar, que deve ser estimulado logo nos primeiros anos de vida, para que a criança mantenha essa rotina quando adulta. Observa-se que as crianças que não tomam café da manhã em casa parecem ser mais propensas a comprar alimentos fora de casa e estes normalmente não são tão saudáveis. ${ }^{10}$

As crianças têm como hábito imitar os pais tanto no que eles fazem, quanto no que comem e, por essa razão os pais que não possuem uma dieta com frutas e verduras, acabam influenciando na alimentação de seus filhos. A prática de tomar o café da manhã, o maior número de refeições diárias, o maior número de refeições em casa e menor número de refeições fora do lar foram considerados como 


\section{JOURNALOF \\ NURSING \\ M०HEALTH}

ISSN 2236 - 1987

pai para filho através das gerações. Os pais que brincam com os seus filhos desde os primeiros anos de vida, os influenciam a manterem uma rotina diária de movimentação corporal. A atividade física é qualquer movimento corporal decorrente de contração muscular, com gasto energético acima do repouso que, em última análise, permite o aumento da força física, flexibilidade do corpo e maior resistência, com mudanças, seja no campo da composição corporal ou de performance desportiva. ${ }^{14}$

As intervenções multiprofissionais com enfoque na redução da obesidade apresentam bons resultados, principalmente quando relacionadas às medidas como peso e índice de massa corpórea, composição corporal e redução de comorbidades, que auxiliam também na melhora do estilo de vida e na autoestima do indivíduo. ${ }^{15}$

A movimentação, sob a forma de atividades lúdicas é de extrema importância para o crescimento saudável de uma criança, visando à continuidade dos hábitos na vida adulta. Algumas crianças entrevistadas citaram a preferência por brincadeiras que não exigem muita movimentação física, e desta forma mantendo-as sedentárias em relação às brincadeiras com movimentação corporal. Podemos identificar que, atualmente as crianças e adolescentes estão cada vez mais substituindo atividades que envolvem algum esforço físico pelas novas tecnologias. Este estilo de vida provavelmente influenciará no estilo de vida adulto. Tal fato é extremamente preocupante, pois o sedentarismo está intimamente relacionado ao fator 


\section{JOURNALOF \\ NURSING \\ ANO HEALTH}

ISSN 2236 - 1987

Uma das estratégias que 0 enfermeiro pode lançar mão são as práticas educativas que envolva a interação com os indivíduos ou grupos na perspectiva de conhecer suas práticas de saúde e o contexto no qual as ações são definidas e, para isso os profissionais da área da saúde, precisam construir novas práticas de cuidado, com participação dos usuários, sendo estes os protagonistas do cuidado interdisciplinar, qualificado na busca da resolutividade e integralidade do cuidado. ${ }^{19-20}$

\section{CONSIDERAÇÕES FINAIS}

A partir dos dados evidenciados neste estudo podem-se conhecer os hábitos cotidianos de alimentação e prática de atividades físicas de crianças entre sete e dez anos de idade, de uma unidade básica de educação de um município do Sul de Santa Catarina. Onde se percebeu que os hábitos de vida saudável destas crianças se constituem em uma situação bastante preocupante, e até mesmo emergencial, que precisa sofrer intervenção imediata, pois nos relatos apareciam que em suas refeições estavam contemplados alimentos de alto teor de açucares e gorduras, além de suas atividades físicas e de lazer também estavam comprometidas com o sedentarismo.

Assim, parcerias entre o ESF, escola e família são importantes para traçar estratégias e fomentar as práticas de educação em saúde desta comunidade. Assim, pode-se ressaltar a importância da realização de atividades, visando à prevenção da obesidade infantil, já que a mesma 


\section{JOURNALOF \\ NURSING \\ ${ }^{\text {ANO }} \mathrm{HEALTH}$ \\ ISSN 2236 - 1987}

tem aumentado gradativamente em nossa população nos últimos anos.

Adotar medidas para obter uma alimentação saudável e realizar a prática de atividades físicas são de fundamental importância para se adquirir hábitos e qualidade de vida saudáveis, ampliando a percepção de que ter uma vida saudável, não somente no presente, mas também no futuro. Tal fato demonstra a importância da atuação do profissional enfermeiro na educação nutricional das crianças, baseados no respeito a cultura da comunidade, buscando a melhoria e promoção dos hábitos alimentares e nas práticas de atividades físicas na infância na vida adulta.

Como limitação deste estudo destaca-se a sutiliza dos dados, visto que este estudo foi desenvolvido em apenas uma escola. Sugere-se que outros estudos sejam desenvolvidos no sentido de buscar identificar no cotidiano de crianças e suas famílias práticas saudáveis de vida.

\section{REFERÊNCIAS}

1. Ministério da Saúde (BR). Secretaria de Vigilância em Saúde. Secretaria de Atenção à Saúde. Política Nacional de Promoção da Saúde: revisão da portaria MS/GM n ${ }^{\circ} 687$, de 30 de março

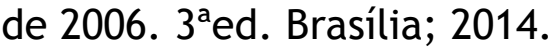

2. Ministério da Saúde (BR). Secretaria de Atenção à Saúde. Departamento de Atenção Básica. Guia alimentar para a população da brasileira. $2^{a}$ ed. Brasília; 2014.

3. World Health organization (WHO). Obesity and overweight. 2016.
4. Associação Brasileira para o Estudo da Obesidade e da Síndrome Metabólica. Diretrizes brasileiras de obesidade. $4^{\mathrm{a}} \mathrm{ed}$. São Paulo: ABESO; 2016

5. Franks PW, Hanson RL, Knowler WC, Siviers ML, Bennett PH, Looker HC. Childhood obesity, other cardiovascular risk factors, and premature death. $\mathrm{N}$ engl $\mathrm{j}$ med. 2011; 362(6):485-93.

6. George JB. Teorias de Enfermagem: os fundamentos para a prática profissional. $1^{\mathrm{a} e d}$. Porto Alegre: Artes Médicas; 1993.

7. Bardin L. Análise de conteúdo. $4^{\mathrm{a}}$ ed. São Paulo: Edições 70; 2011.

8. Camargo JMTB, Marín-León L. Factors associated with overweight among elementary schoolchildren in Campinas, São Paulo, Brazil. Rev nutr. 2016; 29(3): 401-13.

9. Fechine ADL, Machado MMT, Lindsay AC, Fechine VAL, Arruda CAM. Percepção de pais e professores sobre a influência dos alimentos industrializados na saúde infantil. Rev bras promoc saúde. 2015; 28(1):16-22.

10. Utter J, Scragg R, Mhurchu CN, Schaaf D. At-home breakfast consumption among new zealand children: associations with body mass index and related nutrition behaviors. $\mathrm{J}$ am diet assoc. 2007; 107(4):570-6.

11. Prado BG, Hinnig PF, Tanaka LF, Latorre MRDO. Qualidade da dieta de escolares de 7 a 10 anos do município de São Paulo: associação com o número e os locais de refeições. Rev nutr. 2015; 28(6):607-18. 


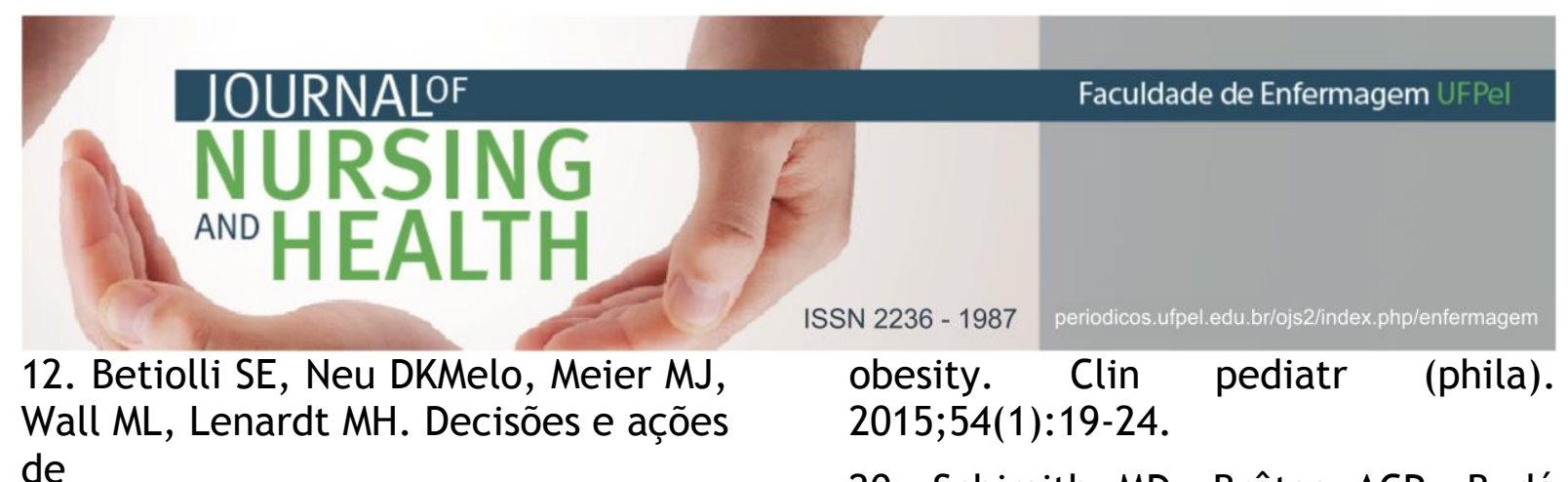
de

cuidados em enfermagem alicerçadas em Madeleine Leininger. Cogitare enferm.

2013;18(4):775-81.

13. Camozzi ABQ, Monego ET, Menezes IHCF, Silva PO. Promoção da Alimentação Saudável na Escola: realidade ou utopia?. Cad. Saúde Colet., 2015;23 (1): 32-7

20. Schimith $M D$, Brêtas $A C P$, Budó MLD, Simon BS, Brum DJT, Barrios SG, et al. Interface entre os diferentes níveis de atenção à saúde: o desafio da complementaridade. J nurs health. 2014;4(2):165-76.

Data de submissão: 03/04/2017

Data de aceite: 16/10/2017

Data de publicação: 26/12/2017

14. Carvalho IL, Carneiro MLM, Reis TC, Pinho L. Estado nutricional de escolares da rede pública de ensino. Rev rene. 2014;15(2):291-7.

15. Ramos AT. Atividade física: diabéticos, gestantes, terceira idade, crianças e obesos. $1^{\text {a }}$ ed. Rio de Janeiro: Sprint; 1999.

16. Bianchini JAA, Hintze JL, Bevilaqua CA, Agnolo CMD, Junior NN. Tratamento da obesidade: revisão de artigos sobre intervenções multiprofissionais no contexto brasileiro. Arq ciênc saúde. 2012;19(2):9-15.

17. Vasconcellos MB, Anjos LA, Vasconcellos MTL. Estado nutricional e tempo de tela de escolares da rede pública de ensino fundamental de Niterói, Rio de Janeiro, Brasil. Cad saúde pública. 2013; 29(4):713-22.

18. Santos FDR, Vitola CB, Arrieira ICO, Chagas MCS, Gomes GC, Pereira FW. Ações de enfermeiros e professores na prevenção e no combate à obesidade infantil. Rev rene. 2014;15(3):463-70.

19. Fox CK, Marlatt KL, Rudser KD, Kelly AS. Topiramate for weight reduction in adolescents with severe 